\title{
Long-chain polyunsaturated fatty acids in breast-milk and erythrocytes and neurodevelopmental outcomes in Danish late-preterm infants
}

\author{
Andersen, Stine Brøndum; Hellgren, Lars I; Larsen, Mette Krogh; Verder, Henrik; Lauritzen, Lotte
}

Published in:

Journal of Pregnancy and Child Health

Link to article, DOI:

10.4172/2376-127x.1000160

Publication date:

2015

Document Version

Publisher's PDF, also known as Version of record

Link back to DTU Orbit

Citation (APA):

Andersen, S. B., Hellgren, L. I., Larsen, M. K., Verder, H., \& Lauritzen, L. (2015). Long-chain polyunsaturated fatty acids in breast-milk and erythrocytes and neurodevelopmental outcomes in Danish late-preterm infants. Journal of Pregnancy and Child Health, 2(3), [160]. https://doi.org/10.4172/2376-127x.1000160

\section{General rights}

Copyright and moral rights for the publications made accessible in the public portal are retained by the authors and/or other copyright owners and it is a condition of accessing publications that users recognise and abide by the legal requirements associated with these rights.

- Users may download and print one copy of any publication from the public portal for the purpose of private study or research.

- You may not further distribute the material or use it for any profit-making activity or commercial gain

- You may freely distribute the URL identifying the publication in the public portal 


\title{
Long-Chain Polyunsaturated Fatty Acids in Breast-Milk and Erythrocytes and Neurodevelopmental Outcomes in Danish Late-Preterm Infants \\ Stine Brøndum Andersen ${ }^{1 *}$, Lars I Hellgren ${ }^{2}$, Mette Krogh Larsen ${ }^{3}$, Henrik Verder ${ }^{1}$ and Lotte Lauritzen ${ }^{4}$
}

${ }^{1}$ Department of Paediatrics, Holbaek University Hospital, Smedelundsgade, Holbaek, Denmark

${ }^{2}$ Centre for Biological Sequence Analysis, Department of Systems Biology, Technical University of Denmark, Søltofts Plads bygning, Lyngby, Denmark

+Department of Food Science, AU Foulum, Aarhus University, Blichers Allé 20, Tjele, Denmark

${ }^{4}$ Department of Nutrition, Exercise and Sports, University of Copenhagen, Rolighedsvej, Frederiksberg C, Denmark

\begin{abstract}
Background: The supply of long-chain polyunsaturated fatty acids (LC-PUFA) during pregnancy and early lactation has been shown to affect cognitive development in preterm infants, but the effect on early neurodevelopment
\end{abstract} of late-preterm infants has not yet been examined.

Aim: To examine the fatty acid composition of late-preterm human milk and identify possible associations between infant LC-PUFA status and perinatal as well as 1-year neurobehavioral outcomes.

Methods: Mother's milk and erythrocytes (RBC) were sampled from 53 Danish late-preterm infants (33-36 weeks of gestation) 1 week and 1 month after delivery, and 3 months corrected age. Fatty acid composition was determined by gas-liquid chromatography. Neurodevelopmental outcomes were assessed by the Nicu Network Neurobehavioral Scale (NNNS) at 1 week and 1 month and the Bayley Scales (BSID-III) at 1 year corrected age.

Results: We found that breast-milk content of arachidonic acid (AA) and docosahexaenoic acid (DHA) was similar to reported fatty acid compositions of term human milk. Infant RBC-AA decreased from 1 week to 1 month of age and the size of the decrease was associated with better NNNS-scores at 1 month, specifically on regulation $(p=0.03)$. Infant RBC-AA at 1 month was also associated with a lower 1-year corrected age BSID-III score of receptive language $(p=0.05)$ and fine motor development $(p=0.03)$. Infant RBC-DHA did not decrease significantly after delivery and was not associated with any of the developmental outcomes.

Conclusion: Breast-milk LC-PUFA content was reflected in the RBC LC-PUFA status of the infant. Early RBC-AA status was associated with both early and long-term neurobehavioral development, but not in a consistent way.

Keywords: Late-preterm infant; Long-chain polyunsaturated fatty acid; Neurodevelopment; Breastfeeding; Human milk; Erythrocytes

Abbreviations: AA: Arachidonic Acid; BSID-III: Bayley Scale of Infant Development, Third Edition; DHA: Docosahexaenoic Acid; LA: Linoleic Acid; LC-PUFA: Long-chain Polyunsaturated Fatty Acid; NNNS: Nicu Network Neurobehavioral Scale; PUFA: Polyunsaturated Fatty Acid; QoM: Quality of Movement; RBC: Erythrocyte (Red Blood Cell); SD: Standard Deviation

\section{Introduction}

Long-chain polyunsaturated fatty acids (LC-PUFA), especially docosahexaenoic acid (C22:6n-3, DHA) and arachidonic acid (C20:4n-6, AA), may affect the development of the infant brain as well as the infant growth pattern both before and after delivery [1,2]. These LCPUFAs accumulate in the brain alongside foetal body fat, particularly during the third trimester of pregnancy and the first few months postterm. They are found especially in the synaptic membranes of neurons of the cortical and midbrain grey matter $[1,3]$ in areas responsible for sensory integration, motor control and attention [4]. During the third trimester the foetus receives approximately $50 \mathrm{mg} /\left(\mathrm{kg}^{*}\right.$ day $)$ of total $\mathrm{n}-3$ LC-PUFA and $400 \mathrm{mg} /\left(\mathrm{kg}^{\star}\right.$ day $)$ of total $\mathrm{n}-6$ PUFA [5-7]. AA seems to accumulate to a larger extend than DHA in the foetal brain until around the time of expected delivery; but subsequently DHA is the dominant LC-PUFA $[5,8]$.

Metabolic maturation of cells and organs is believed to take place in the late stage of gestation $[9,10]$ and in the early months after delivery at which time the foetal brain undergoes several structural changes. Neural differentiation, formation and adaptation of synaptic functions as well as glial cell production and myelination take place alongside neuronal regression and conversion of the foetal cortical subplate into a mature cortical structure. This transition is believed to mediate the simultaneous change in infant neuro-behaviour, e.g. in motor control from primitive reflexes and jerky movements to smooth and volitional motion [5,11]. Preterm delivery carries a risk of disrupting these processes, and apparently healthy, late-preterm infants (defined as infants born with a gestational age of 34-36 weeks) can experience long-term developmental problems such as difficulties with attention span, language skills and visuo-spatial motor function [11].

Human milk displays large individual and regional variations in the content of LC-PUFA, most pronouncedly in DHA, which also varies during the course of the day reflecting the content of the individual meals of the mother [12]. Individual factors such as maternal lifestyle, dietary intake and supplementation are determinants for the presence of individual PUFA in the milk [1]. LC-PUFA has been shown to decrease during the lactation period, and breast-milk of mothers with preterm delivery has been shown to have a higher content of LC-PUFA

*Corresponding author: Stine Brøndum Andersen, Department of Paediatrics, Holbaek University Hospital, Smedelundsgade 60, DK-4300 Holbaek, Tel: +45 20254583; E-mail: sjoense@gmail.com

Received: February 13, 2015; Accepted: May 01, 2015; Published: May 06, 2015

Citation: Andersen SB, Hellgren LI, Larsen MK, Verder H, Lauritzen L (2015) Long-Chain Polyunsaturated Fatty Acids in Breast-Milk and Erythrocytes and Neurodevelopmental Outcomes in Danish Late-Preterm Infants. J Preg Child Health 2: 160. doi:10.4172/2376-127X.1000160

Copyright: (c) 2015 Andersen SB, et al. This is an open-access article distributed under the terms of the Creative Commons Attribution License, which permits unrestricted use, distribution, and reproduction in any medium, provided the original author and source are credited. 
[13-16], but the fatty acid composition of late-preterm breast-milk is not well described [1].

To our knowledge, measures of early sensory integration such as stimulus habituation, level of arousal and quality of general movements (QoM) have only been sparsely evaluated as endpoints for LC-PUFA effects or as confounders for later neurodevelopment [17-21]. The aim of the current prospective cohort study was to investigate LC-PUFA status and development in late-preterm infants and to see to what extent measures of early sensory integration and later cognitive performance was associated with infant LC-PUFA status. We hypothesize that latepreterm infants may depend on DHA for optimal neurodevelopment.

\section{Materials and Methods}

\section{Study population}

In the period May 2011 to July 2012, 53 infants born at gestational age of 33.1-36.6 weeks were recruited within a few days after delivery (max 7 days) from the neonatal ward of the Department of Paediatrics, Holbaek University Hospital, which is a minor urban hospital in the provincial part of Zealand, Denmark. Gestational age at delivery was the only inclusion criterion. Exclusion criteria were: severe peripartum asphyxia (standard base excess $<-16$ or transfer to a highly specialized hospital for cooling treatment), severe septicaemia, known serious malformations, distance from residence to the hospital $<2 \mathrm{~h}$ transport, severe psychosocial challenges (e.g. early forced removal of the infant) and major linguistic barriers in the communication with the parents.

The study was approved by The National Committee on Health Research Ethics in Region Zealand (SJ-198) and the Danish Data Protection Agency, and was conducted in accordance with the Declaration of Helsinki. Written informed consent was obtained from both infant custody holders at enrolment.

\section{Clinical examinations}

The recruited infants were examined four times during the first year of life; at 1 week and 1 month after delivery and at 3 and 12 months corrected age (after expected date of delivery). The 1-year follow-up examinations were carried out from June 2012 to July 2013.

Samples of infant blood and mother's milk were collected at the first three visit, and the parents filled out a questionnaire about lactation (extend and duration), formula feeding, maternal and child health, lifestyle, socioeconomic factors and dietary supplementations as well as maternal food-frequency topics. Between the visits the parents were asked to keep a log of infant feeding mode on a weekly basis and in case of any formula feeding to indicate the ratio of formula to breastmilk as well as the brand name of the formula. Exclusive breastfeeding was defined as $<1$ formula meal per week and $<1$ breast-milk meal per day was defined as exclusive formula feeding. Furthermore, the parents were asked to $\log$ the health status and any medication of the child. The project physician and the parents reviewed the logs at every outpatient visit to intercept and record any possible discrepancies. The parents returned the completed logs at the third visit.

\section{Developmental testing}

Infant neuro-developmental status was assessed at 1 week and 1 month after delivery using the Nicu Network Neurobehavioral Scale (NNNS) [10]. NNNS is a neurobehavioral performance test that covers neurological maturation including motor maturity, tone, posture, general autonomic signs of stress and tolerance to stimulation, reflexes, social- and self-regulatory competencies [10]. NNNS scores have been shown to correlate with outcome measures on the Bayley Scales of Infant Development (BSID) [21,22]. NNNS is designed for use within gestational age 30 to approximately $46-48$ weeks and normative preterm and healthy full term samples are available in the test manual [23]. For this study, we selected 3 out of 13 available summary scores as described in the Statistics section below.

Infant developmental milestones were recorded in the period between 3 and 12 months corrected age. Developmental outcomes at 12 months corrected age were determined by the third version of the BSID (III, Danish version 2009, Pearson Assessment, San Antonio, USA). BSID is the long-standing gold standard for infant global developmental testing and a proven method for detecting developmental anomalies. BSID evaluates cognitive, language and motor skills. Receptive and expressive language skills are evaluated separately and combined into an aggregate score and the same applies to fine motor and gross motor skills [24].

Testing conditions for both NNNS and BSID were selected to create the best possibilities for the infants to stay relaxed and attentive. All examinations took place in a quiet ambulatory room without wall decorations. The curtains were closed and the computer screen turned off, and for the NNNS test electric lights were off to keep the infants from focusing on lights and shadows. Both tests were performed halfway between meals if possible and the infants were examined in the presence of only one parent to avoid distraction. Furthermore, we aimed to begin the NNNS examination while the infant was still asleep. All examinations were video recorded, and performed by the lead investigator after proper training by a certified instructor [10].

\section{Blood sampling and fatty acid analysis}

At each visit, blood was drawn in 2-mL lithium-heparin vials. Erythrocytes (RBC) were immediately isolated by centrifugation at $2300 \times \mathrm{G}$ for 5 minutes at $4^{\circ} \mathrm{C}$. Plasma was removed and the $\mathrm{RBC}$ were washed three times in $0.9 \% \mathrm{NaCl}$ with $1 \mathrm{mM}$ EDTA at pH 7.4, resuspended 1:1 in an isotonic $\mathrm{NaCl}$-solution with a final concentration of $0.1 \%$ butylated hydroxytoluen (VWR - Bie \& Berntsen, Herlev, Denmark) and stored under $\mathrm{N} 2$ at $-80^{\circ} \mathrm{C}$ for a maximum of 3 months.

RBC fatty acid composition was determined as previously described [25]. In brief: RBC were haemolysed in redistilled water, and the lipids were extracted by the Folch procedure [26]. Fatty acid methyl esters were produced from the extracted RBC lipids using a BF3-catalyzed method [27] and extracted with heptane and analysed by gas-liquid chromatography on an Agilent 6890 Series II chromatograph (Agilent Inc., Santa Clara, California, USA) equipped with a flame ionization detector and a SP2380 capillary column (length $60 \mathrm{~m}$; internal diameter $0.25 \mathrm{~mm}$ and film thickness $0.2 \mu \mathrm{m}$ from Supelco Inc, Pennsylvania, USA) [28]. Fatty acid peaks were identified using a standard mixture (Nu-Check Prep, Elysian, $\mathrm{MN}$ ), and data are given as mass-\% based on peak-area relative to the sum of all peak areas corrected for differences in detector-response. The average intra-assay coefficient of variation was $3.0 \%$ for linoleic acid (LA), $0.4 \%$ for eicosapentaenoic acid and $0.2 \%$ for AA and DHA, and the inter-assay coefficient of variation were $4 \%$ for LA, $0.6 \%$ for eicosapentaenoic acid and $0.4 \%$ for AA and DHA.

\section{Milk sampling and fatty acid analysis}

A $50 \mathrm{~mL}$ breast-milk sample was collected between 5 and 8 a.m. in the last days up to each examination using an electric hand-held breast pump (Medela Swing, Medela AG, Baar, Switzerland). Many mothers could not deliver the $50 \mathrm{~mL}$ at once, especially at the first sampling, so they were allowed up to three consecutive mornings to collect 
the sample. Mothers were instructed to freeze their milk samples immediately after pumping in their home freezer, and in case they had to do the pumping on more than one morning, to keep the samples in separate containers. The mothers transported the milk samples from their home freezer to the clinic in an UV-proof black cooler bag containing a freezing element with capacity to keep the bag content frozen for the duration of the drive to the clinic. Immediately upon arrival, milk samples were placed at $-18^{\circ} \mathrm{C}$ until further processing. Within a few days ( $\leq 7$ days), the samples were thawed in a $20^{\circ} \mathrm{C}$ water bath; sub-samples were pooled and gently mixed. The samples were aliquoted in $0.5 \mathrm{~mL}$ containers and $0.1 \%$ butylated hydroxytoluen in ethanol was added to aliquots for fatty acid analysis. The samples were then blown with $\mathrm{N} 2$ and kept at $-80^{\circ} \mathrm{C}$ until analysis. Samples were analysed within 3 months from collection.

The breast-milk samples were freeze-dried for analysis of fatty acid composition. The freeze-dried material was mixed with $1 \mathrm{~mL}$ of heptane containing $0.4 \mathrm{mg} / \mathrm{mL}$ of internal standard (C12:1 cis11 triacylglycerol from NuChekPrep Inc, Elysian MN, USA). Methylation was carried out as a two-step procedure based on Jenkins [29] with modifications. Base-catalysed methylation carried out by addition of $0.2 \mathrm{~mL} 25 \%$ sodium methoxide, followed by incubation at $50^{\circ} \mathrm{C}$ for 10 min. After cooling $1.5 \mathrm{~mL}$ of a $10 \%$ methanolic $\mathrm{HCl}$ was added and the samples were incubated at $90^{\circ} \mathrm{C}$ for $30 \mathrm{~min}$. After cooling $3 \mathrm{~mL}$ $10 \% \mathrm{~K} 2 \mathrm{CO} 3$ was added, tubes were centrifuged and the heptane layer was isolated. Separation of the fatty acid methyl esters was carried out on an Agilent 6890 gas chromatograph with a flame ionization detector and a Restek 2560 capillary column (length $100 \mathrm{~m}$, internal diameter $0.25 \mathrm{~mm}$ and film thickness $0.20 \mu \mathrm{m}$ from Restek, Bellefonte, Pennsylvania, USA). The temperature program started at $100^{\circ} \mathrm{C}$ for 5 min, followed by an increase of $3^{\circ} \mathrm{C} / \mathrm{min}$ to $140^{\circ} \mathrm{C}$, another increase of $20^{\circ} \mathrm{C} / \mathrm{min}$ to $160^{\circ} \mathrm{C}$, where it was kept for $20 \mathrm{~min}$ followed by an increase of $12^{\circ} \mathrm{C} / \mathrm{min}$ to $220^{\circ} \mathrm{C}$, which was then kept for $15 \mathrm{~min}$ after which it was increased at $8^{\circ} \mathrm{C} / \mathrm{min}$ to a final temperature of $240^{\circ} \mathrm{C}$ for $8 \mathrm{~min}$. The carrier gas was helium at a constant flow of $1 \mathrm{~mL} / \mathrm{min}$. The individual peaks were identified by use of external standards (37 component mix from Supelco, Belafonte, USA and GLC 469 standard from NuChekPrep Inc., Elysian, MN, USA) and quantified by use of the internal standard. The total sum of fatty acids was calculated as $\mathrm{g} / \mathrm{L}$ milk and the content of individual fatty acids was expressed as mass- $\%$ fatty acids. The coefficients of variation for LA, AA and DHA were $4 \%$, $5 \%$ and $5 \%$, respectively.

\section{Statistics}

The statistical analyses were performed by IBM SPSS Statistics software (version 20.0). Data are presented as frequencies or mean \pm SD. Students t-test was used for group comparisons e.g. gender differences and participants vs. non-participants (excluded infants, those refusing to participate and those lost to follow-up). Correlations between exposures, outcomes and possible covariates were calculated using the non-parametric Spearman's test. Assumption of normality was tested by Shapiro-Wilk's test and visual inspection of histograms, and by examining the residual plots of the final univariate linear models.

Univariate general linear models were used to test associations between LC-PUFA and developmental scores. The models were built by backwards-stepwise exclusion $(\mathrm{p}<0.05)$ of possible covariates: gender, gestational age at delivery, maternal education, socioeconomic status (composite score of education and occupation as defined by The Danish National Centre for Social Research) maternal psychiatric disorder (e.g. depression), multiple pregnancy, smoking in pregnancy, maternal multiparity and age at neurological assessment (days). None of these covariates were correlated (Spearman $\mathrm{p}>0.05$ ). Furthermore, maternal smoking was also tested as a potential covariate for breastmilk LC-PUFA levels.

To avoid multiple testing we carefully selected the measures of breast-milk and infant RBC LC-PUFA as well as the NNNS scores to be used in analyses of association. In order to do that we used information about breastfeeding from questionnaires and interviews and data on the fatty acid composition of the breast-milk and infant RBC samples to determine which samples to use in our correlation and association analyses. The individual variation in breast-milk DHA (1.03\% at 1 week and $1.35 \%$ at 1 month) exceeded the changes over time, i.e. between the means at 1 week and 1 month $(0.19 \%)$, so we estimated that the mean DHA of all three samples would give the most accurate description of infant DHA intake during the breastfeeding period. We tested this assumption by comparing the mean after 1 month with the combined mean (by t-test); and as we found no difference, we chose the combined mean as our independent variable in the subsequent analyses. The same rationale applied to breast-milk AA.

Infant RBC LC-PUFA levels are expected to be associated with brain accumulation of AA and DHA [30] so we considered infant RBC levels the best proxy for brain LC-PUFAs and chose RBC as our main focus in the analysis of association with infant neurodevelopment. The following rationale was applied in selecting which RBC samples to use in our analyses: Because of the approximately 3-months turnover of the RBC, the 1-week infant RBC sample reflects the LC-PUFA supply during pregnancy and does not reflect supply from breastfeeding. The 1-month sample reflects the LC-PUFA supply from 32 weeks of gestation to 4 weeks post-term, and thus covers 8-12 weeks of extrauterine enteral feeding depending on the infant's gestational age at delivery, whereas the sample taken at 3 months corrected age in teory should reflect the entire early feeding period. However, as descriptive statistics showed an increasing uniformity of the 3 months samples and a discrepancy between the 3 months sample and the previous two samples and as an increasing number of children stopped breastfeeding, we considered the sample taken at 1 month post-term to be the best measure of infant LC-PUFA uptake from breast-milk.

NNNS generates 13 summary scores, some of which measure primarily physiological instability or neurological damage, which was outside the scope of this study (excitability, lethargy, stress/abstinence, handling, hyper-/hypotonia [31]). To find the scores most suitable to use for comparison of development between infants, we reviewed the internal correlation of scores over time (by Spearman's test), number of missing values, and normal distribution as well as how the scores reflected hypotheses in previous studies in the LC-PUFA field. Three out of the remaining 7 summary scores were used in our analyses: quality of movement (QoM), regulation and arousal (which was strongly negatively correlated with habituation, $\mathrm{r}=-0.42, \mathrm{p}=0.006$ ). QoM summarizes motor maturity, control and activity as well as the ability to perform smooth movements without startles, tremors or jitters. Regulation evaluates the combined scores of attention, motor and psychological activity and the infant's capacity to regain comfort with and without examiner assistance. Arousal is a measure of the infant's overall level of motor activity and irritability, fuss and cry in response to handling, as well as the state of arousal reached during the examination [32]. Higher scores in all three variables indicate a higher degree of neuro-maturation.

We examined if RBC LC-PUFA status during the perinatal period was associated with the three NNNS measures at delivery and at 1 
month post-delivery by correlation analysis and univariate linear regression models adjusted for gender and gestational age. BSID-III scores at 1 year corrected age (cognitive, receptive language, expressive language, fine motor and gross motor scale scores) were also tested against AA and DHA in breast-milk and infant RBC. To determine whether neuro-developmental status at delivery excerts a confounding influence on developmental status in later infancy, we included the NNNS measures as covariates in the final models for the BSID-III outcomes.

Due to the explorative nature and the small sample size of this study, performing a rigorous correction for multiple testing (e.g. by Bonferroni) would increase the risk of type-II errors considerably. We therefore chose not to correct for multiple testing, but to report the p-values of our analyses and to discuss the potential relevance of findings with $\mathrm{p}$-values $<0.05$.

\section{Results}

The inclusion rate was $69 \%$ (Figure 1). The characteristics of the included mothers and infants (Table 1) differed only from nonparticipants by slightly younger mothers $(29 \pm 5$ years vs. $31 \pm 4$ years, $\mathrm{p}=0.048)$. The rate of loss to follow-up was $9 \%(\mathrm{n}=5)$ and the lost infants differed from the remaining infants by a higher rate of maternal psychiatric disorder $(p=0.015)$, although this was caused by only one twin mother $(\mathrm{n}=2)$.

Sixteen infants (30\%) were never exclusively breastfed and introduction of complementary feeding was initiated at a mean age of $147 \pm 25$ days corresponding to $116 \pm 24$ days post-term. Infants who were breastfed at 3 months corrected age differed from those who were not by the frequency of maternal smoking in pregnancy ( $50 \%$ smokers in the non-breastfeeding group vs. $5.4 \%$ in the breastfeeding group, $\mathrm{p}<0.001)$ and maternal smoking was correlated with low socioeconomic status $(\mathrm{r}=0.29, \mathrm{p}=0.035)$.

\section{Fatty acid composition of breast-milk and infant RBC}

The LC-PUFA composition of the breast-milk and the infant RBC samples are shown in Table 2. There was a significant $26 \%$ decline $(p<0.001)$ in breast-milk DHA during the first month, but there was

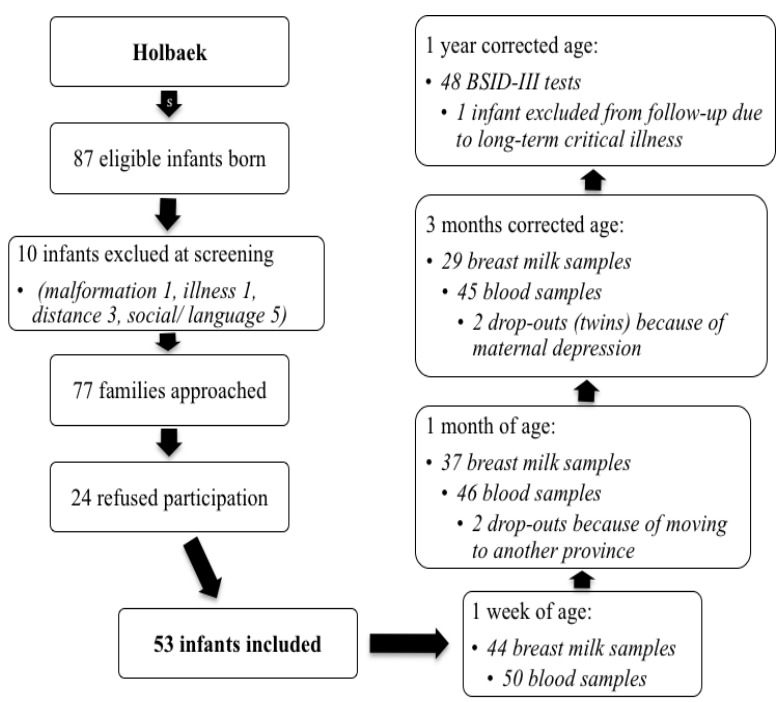

Figure 1: Participants. Flow of inclusion and exclusion, and of visits and sample collection. no significant difference between the fatty acid composition of the milk samples at 1 month after delivery and at 3 months corrected age. The mean levels of AA and DHA in the combined samples were $0.61 \pm 0.13$ mass- $\%$ for AA and $0.66 \pm 0.28$ mass- $\%$ for DHA. The RBC content of LA and DHA in the infants increased over time, whereas that of AA decreased (all changes $\mathrm{p}<0.05$ ).

Overall, the correlations between breast-milk and infant RBC were positive for both AA and DHA (Table 3). Breast-milk and RBC-LA were only correlated at 1 month after delivery. There was no associaton between gestational age at delivery and infant RBC-DHA, AA or LA, although AA showed a trend towards a negative association $(B=-$ $0.07 \pm 0.032, p=0.051)$. There was a decrease in the variance for infant $\mathrm{RBC}-\mathrm{DHA}$ and $\mathrm{AA}$ at 3 months corrected age, and the correlations between the infant RBC samples at 1 month and 3 months as well as between breast-milk and infant RBC at 3 months were not significant except for AA (Table 3), which may be due to the increase in formula feeding frequency after 1 month of age.

\section{LC-PUFA and NNNS}

Paired t-tests between the NNNS scores at 1 week and 1 month revealed that regulation and arousal increased over time, but no increase was seen in QoM (Table 5A). QoM at 1 week and 1 month of age were found to correlate (Spearman $\mathrm{r}=-0.31, \mathrm{p}=0.02$ ), whereas no correlations were seen for regulation $(\mathrm{r}=0.02, \mathrm{p}=0.90)$ and arousal $(\mathrm{r}=0.12, \mathrm{p}=0.39)$. There was no gender difference in the NNNS scores at any of the examinations.

There were no significant correlations (Spearman) between the levels of AA or DHA in infant RBC at 1 month of age and any of the NNNS scores. There was also no correlation between the changes in infant RBC-AA and DHA between 1 week and 1 month and the corresponding changes in the NNNS scores, but arousal at 1 month of age tended to correlate negatively with the change in infant RBC-AA $(\mathrm{r}=-0.30, \mathrm{p}=0.050)$. The relationships between infant RBC LC-PUFA status and the NNNS neuro-developmental measures at 1 week and 1 month were also examined by analyses adjusted for gender and gestational age at delivery (Table 5B). At 1 week of age we found no significant associations between the NNNS scores and RBC LCPUFA, but infant RBC-AA was found to be positively associated with regulation 1 month after delivery (Table $5 \mathrm{~B}$ ). This association was also significant after adjusting for AA status at 1 week $(\mathrm{p}=0.028)$. Adjusting for regulation at 1 week of age had no impact on this association. Infant RBC-DHA at 1 month or the change over time in RBC-DHA was not associated with any of the NNNS scores at 1 month. The changes in NNNS scores between the two examinations and the corresponding changes in RBC-AA and DHA were not associated.

\section{LC-PUFAs and BSID-III}

T-tests revealed a gender difference in the score on the cognitive scale with girls having $14 \%$ higher scores than boys $(\mathrm{p}=0.045)$. No other gender differences were found in the BSID-III scores. The correlations between the breast-milk sample LC-PUFA means and infant RBC LC-PUFA at 1 month of age versus BSID-III outcomes were generally not significant, except for breast-milk AA, which correlated positively with the cognitive scale score $(\mathrm{p}=0.002, \mathrm{r}=0.46)$. The mean AA for all breast-milk samples was also associated with the cognitive score in models adjusted for gender $(B=8.69 \pm 3.08, p=0.007)$. Furthermore, $\mathrm{RBC}-\mathrm{AA}$ was associated with the receptive language and fine motor scale score in univariate adjusted GLM models, but in this it was an inverse association (Table 6). Inclusion of infant NNNS scores at 1 week as covariates did not change the associations between RBC LC- 
Citation: Andersen SB, Hellgren LI, Larsen MK, Verder H, Lauritzen L (2015) Long-Chain Polyunsaturated Fatty Acids in Breast-Milk and Erythrocytes and Neurodevelopmental Outcomes in Danish Late-Preterm Infants. J Preg Child Health 2: 160. doi:10.4172/2376-127X.1000160

Page 5 of 9

\begin{tabular}{|c|c|c|c|c|c|c|}
\hline & \multicolumn{3}{|c|}{ Male $(n=32)$} & \multicolumn{3}{|c|}{ Female $(n=21)$} \\
\hline & $\%$ yes & Mean \pm SD & Range & $\%$ yes & Mean \pm SD & Range \\
\hline Post-mentrual age, days & & $248 \pm 8$ & $233-259$ & & $248 \pm 8$ & $231-258$ \\
\hline Birth weight, g & & $2503 \pm 456$ & $1346-3235$ & & $2655 \pm 530$ & $1455-3930$ \\
\hline Mother's age, yrs & & $30 \pm 6$ & $20-40$ & & $27 \pm 3$ & $20-31$ \\
\hline Pre-pregnancy weight, kg & & $66 \pm 13$ & $49-95$ & & $72 \pm 13$ & $47-98$ \\
\hline Pre-pregnancy BMI, kg/m² & & $24.6 \pm 4.6$ & $18.9-37.1$ & & $24.8 \pm 4.1$ & $16.7-32.9$ \\
\hline Weight gain in pregnancy, kg & & $14 \pm 6$ & $1-29$ & & $14 \pm 5$ & $6-21$ \\
\hline Smoking in pregnancy & 15.6 & & & 19.0 & & \\
\hline Multiple birth & 31.3 & & & 19.0 & & \\
\hline Low socioeconomic status ${ }^{1}$ & 40.6 & & & 28.6 & & \\
\hline Maternal psychiatric disorder & 25.0 & & & 42.9 & & \\
\hline Any breastfeeding, wks & & $33 \pm 22$ & $0-60$ & & $26 \pm 21$ & $0-60$ \\
\hline Exclusive breastfeeding, wks & & $6 \pm 5$ & $0-12$ & & $7 \pm 6$ & $0-12$ \\
\hline Prop. of breastfeeding, \% & 1 week & 1 month & 3 months $^{2}$ & 1 week & 1 month & 3 months $^{2}$ \\
\hline Exclusive breastfeeding & 31.3 & 28.1 & 62.5 & 38.1 & 38.1 & 61.9 \\
\hline Combination & 62.5 & 62.5 & 6.3 & 57.1 & 52.4 & 9.5 \\
\hline Exclusive formula & 6.3 & 6.3 & 28.1 & 4.8 & 4.8 & 23.8 \\
\hline Missing & 0 & 3.1 & 3.1 & 0 & 4.8 & 4.8 \\
\hline
\end{tabular}

Data are given as mean $\pm S D$ and range minimum-maximum or percent as appropriate.

${ }^{1}$ As defined by The Danish National Centre for Social Research

${ }^{2}$ Corrected age

Table 1: Characteristics of the study population by infant gender.

\begin{tabular}{|c|c|c|c|c|c|}
\hline & Sampling time & 1 week & 1 month & 3 months corrected age & Effect of sample no \\
\hline & Infant age (days) & $9.40 \pm 3.03$ & $31.90 \pm 4.53$ & $93.80 \pm 5.76$ & \\
\hline \multirow{11}{*}{$\begin{array}{l}\text { O } \\
\text { r }\end{array}$} & & $n=50$ & $n=46$ & $n=45$ & \\
\hline & Fatty acid & & & & $\mathrm{p}$ (explained var) \\
\hline & LA & $6.42 \pm 0.91$ & $8.74 \pm 1.23$ & $9.34 \pm 1.18$ & $\uparrow 0.00(56.8 \%)$ \\
\hline & AA & $18.44 \pm 1.76$ & $16.99 \pm 1.72$ & $17.34 \pm 1.47$ & $\downarrow 0.00(17.2 \%)$ \\
\hline & DHA & $6.88 \pm 0.96$ & $6.59 \pm 0.87$ & $7.03 \pm 1.04$ & $\uparrow 0.03(7.8 \%)$ \\
\hline & Total SFA & $38.78 \pm 1.46$ & $37.89 \pm 2.26$ & $36.07 \pm 1.67$ & \\
\hline & Total MUFA & $15.76 \pm 1.02$ & $16.70 \pm 1.17$ & $15.99 \pm 1.43$ & \\
\hline & Total n-3 PUFA & $8.17 \pm 1.15$ & $8.21 \pm 1.14$ & $9.43 \pm 1.24$ & \\
\hline & Total n-6 PUFA & $32.03 \pm 2.02$ & $32.93 \pm 2.07$ & $28.20 \pm 1.35$ & \\
\hline & Total PUFA & $40.20 \pm 2.37$ & $41.14 \pm 2.59$ & $37.63 \pm 1.69$ & \\
\hline & n-6/n-3 PUFA ratio & $3.99 \pm 0.61$ & $4.08 \pm 0.58$ & $3.05 \pm 0.46$ & \\
\hline & & $\mathrm{n}=44$ & $\mathrm{n}=37$ & $\mathrm{n}=29$ & $\mathrm{p}$ (explained var) \\
\hline \multirow{10}{*}{ 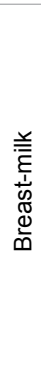 } & LA & $10.76 \pm 2.78$ & $10.78 \pm 1.95$ & $11.13 \pm 1.78$ & $0.85(0.6 \%)$ \\
\hline & $\mathrm{AA}$ & $0.72 \pm 0.17$ & $0.52 \pm 0.11$ & $0.49 \pm 0.11$ & $\downarrow 0.00(56.3 \%)$ \\
\hline & $\mathrm{DHA}$ & $0.74 \pm 0.24$ & $0.55 \pm 0.27$ & $0.64 \pm 0.61$ & $0.09(8.3 \%)$ \\
\hline & Total SFA & $44.74 \pm 6.36$ & $44.52 \pm 4.78$ & $43.80 \pm 7.34$ & \\
\hline & Total MUFA & $39.51 \pm 4.74$ & $40.36 \pm 3.86$ & $40.85 \pm 6.16$ & \\
\hline & Total n-3 PUFA & $2.59 \pm 0.56$ & $2.43 \pm 0.70$ & $2.55 \pm 1.06$ & \\
\hline & Total n-6 PUFA & $12.08 \pm 2.85$ & $11.87 \pm 1.96$ & $12.11 \pm 1.84$ & \\
\hline & Total PUFA & $14.67 \pm 3.16$ & $14.30 \pm 2.40$ & $14.65 \pm 2.38$ & \\
\hline & n-6/n-3 PUFA ratio & $4.76 \pm 1.10$ & $5.16 \pm 1.23$ & $5.35 \pm 1.90$ & \\
\hline & Total fat $(\mathrm{g} / \mathrm{L})$ & $34.6 \pm 13.0$ & $36.8 \pm 11.7$ & $30.6 \pm 15.7$ & \\
\hline
\end{tabular}

Data are given as mean $\pm S D$. $L A=$ linoleic acid. $A A=$ arachidonic acid. $D H A=$ docosahexaenoic acid.

SFA=saturated fatty acid. MUFA=monounsaturated fatty acid. PUFA=polyunsaturated fatty acid.

Table 2: Fatty acid composition in breast-milk and infant erythrocytes.

PUFA and BSID-III scores. These models did not show any significant associations between the NNNS scores at 1 week and the BSID-III scores at 1 year, except for QoM which was associated with expressive language $(\mathrm{B}=1.35 \pm 0.63, \mathrm{p}=0.040)$.

\section{Discussion}

Our most important findings were that $\mathrm{AA}$ and DHA in the breast-milk and infant RBC samples were significantly associated and that both early and later neurodevelopment of late-preterm infants was not related to RBC-DHA, but related to infant RBC-AA status. The fatty acid composition of the breast-milk samples demonstrated a considerable individual variation. Compared to the present study, previous Danish and Dutch studies [26,34] found lower breast-milk levels of AA and DHA, and similar levels of LA in mothers of term infants with low-average fish intake. These studies also reported a reduction in the breast-milk content of AA and DHA from delivery 
to later stages of breastfeeding as well as an increase in the LA content $[26,34]$. Two Swedish studies on moderately-late preterm infants have also shown lower breast-milk levels of all three PUFAs [5,34], but a Spanish study found a considerably higher breast-milk levels of LA and AA levels, which were similar to the ones we observed [13]. The Spanish study did not include any breast-milk samples from late-stage lactation, but found that the DHA level increased over the first few weeks of lactation from initially $0.1 \%$ to $0.5 \%$ of the fatty acids [13]

The individual levels of both LA, AA and DHA in the RBC from the infants in the present study tended to be higher than that reported in more preterm populations [35-37], but resembled reported levels in RBC from both Danish [25] and American term infants [38,39]. The fatty acid composition of the infant RBC at 1 month of age was found to reflect that a considerable proportion of the infants were to some extent formula fed. All formulas on the Danish market contain LA, AA and DHA, although in varying amounts. The infants in our cohort were supplemented according to local guidelines with either a hydrolysed formula with high levels of LC-PUFA (Nutramigen-1 Lipil from

\begin{tabular}{|c|c|c|c|c|}
\hline & Sampling time & LA & AA & DHA \\
\hline \multirow{3}{*}{$\begin{array}{c}\text { Breast-milk } \\
\text { vs. } \\
\text { RBC }\end{array}$} & 1 week & NS & $\begin{array}{l}p=0.04 \\
r=0.33\end{array}$ & $\begin{array}{l}p=0.03 \\
r=0.35\end{array}$ \\
\hline & 1 month & $\begin{array}{l}\mathrm{p}=0.00 \\
\mathrm{r}=0.51\end{array}$ & $\begin{array}{l}p=0.00 \\
r=0.51\end{array}$ & $\begin{array}{l}p=0.00 \\
r=0.48\end{array}$ \\
\hline & 3 months $^{1}$ & NS & $\begin{array}{l}p=0.03 \\
r=0.44\end{array}$ & NS \\
\hline
\end{tabular}

Data are given as Spearman's correlation coefficient $(r)$ and $p$-value. NS=nonsignificant. $L A=$ linoleic acid.

$A A=$ arachidonic acid. $D H A=$ docosahexaenoic acid. $R B C=$ erythrocyte.

${ }^{1}$ Corrected age

Table 3: Correlations between fatty acids in breast-milk and infant erythrocytes (RBC).

\begin{tabular}{|l|c|c|c|c|}
\hline \multirow{2}{*}{} & \multicolumn{2}{|c|}{ Male (n=30) } & \multicolumn{2}{c|}{ Female (n=18) } \\
\cline { 2 - 5 } & Mean \pm SD & Range & Mean \pm SD & Range \\
\hline Cognitive scale score & $12.0 \pm 3.1$ & $5-18$ & $13.7 \pm 2.1$ & $10-17$ \\
\hline Receptive scale score & $8.9 \pm 2.4$ & $5-15$ & $10.3 \pm 3.1$ & $6-16$ \\
\hline Expressive scale score & $10.5 \pm 2.0$ & $7-15$ & $10.8 \pm 1.9$ & $8-16$ \\
\hline Fine motor scale score & $11.5 \pm 2.4$ & $8-16$ & $12.7 \pm 3.6$ & $7-19$ \\
\hline Gross motor scale score & $8.7 \pm 2.0$ & $4-13$ & $9.6 \pm 2.8$ & $3-15$ \\
\hline
\end{tabular}

Data are given as mean $\pm S D$ and range (min-max).

Table 4: Developmental scores on Bayley Scales of Infant Development-III at 1 year corrected-age.
Mead Johnson Nutrition) or standard formulas with moderate levels of LC-PUFA (e.g. NAN-1 from Nestlé or Allomin-1 from Semper). Breastfeeding mothers were potentially biased by their participation in the study to consume more fish and the high levels of LC-PUFA in the RBC may therefore be influenced by a higher-than-average LC-PUFA intake. A larger variation in the RBC LC-PUFA levels would have been better in terms of showing relationships between LC-PUFA status and neurodevelopment.

A few previous studies have found that LC-PUFA composition of breast-milk/formula, maternal RBC and umbilical cord blood was associated with the early QoM in term infants [18-20] as well as in preterm infants (born in gestational week 24-36) [17,35], but the investigated LC-PUFA indices and ratios vary and the results are not very consistent. None of these studies have tested the infants as shortly after delivery as in the present study. However, we found no associations between LC-PUFA and the NNNS scores at 1 week. Our results did however show a positive association between infant RBC-AA (both at 1 week and 1 month) and the NNNS score for regulation at 1 month of age. RBC-AA at 1 month of age was also found to be negatively associated with BSID-III receptive language and fine motor abilities at 1 year corrected age, but no associations were observed for DHA. The lack of a significant associations between breast-milk or RBC-DHA and developmental outcome are in line with previous observations [40-42], although some studies have demonstrated beneficial effects of DHA on psychomotor development in both term and preterm infants [35,43-45]. A recent Swedish study found significant correlations between breast-milk AA and developmental outcomes at 40 weeks gestational age in preterm infants (born in gestational week 24-36) using the Brazelton Neonatal Behavioral Assesment Scale, which is very similar to the NNNS [16]. Another Swedish cohort study [34] of moderately preterm infants found positive associations between both AA and DHA in infant blood and global developmental outcomes at 18 months of age, but negative associations with $n-6$ PUFA and the n-6/n-3 PUFA ratio. The observed assocition between AA and language skills is in accordance with some previous studies of term infant showing negative associations between LC-PUFA intake or status and language development $[26,46]$, but other studies found no effect $[47,48]$. The previous studies have primarily focused on the effect of supplementation with fish oil, DHA or DHA+AA. Recent large randomized controlled trials have shown a negative effect of maternal DHA supplementation in pregnancy on the language development in girls born at term [48], but a positive effect of post-delivery DHA

\begin{tabular}{|c|c|c|c|c|c|c|c|c|c|c|}
\hline \multirow{2}{*}{\multicolumn{2}{|c|}{ A }} & \multicolumn{3}{|c|}{1 week } & \multicolumn{3}{|c|}{1 month } & \multicolumn{3}{|c|}{$\begin{array}{c}\text { Change over time } \\
\text { ( } \Delta \text { NNNS })\end{array}$} \\
\hline & & Mean \pm SD & \multicolumn{2}{|c|}{ Range } & Mean \pm SD & \multicolumn{2}{|c|}{ Range } & Mean \pm SEM & $\mathrm{p}$ & $\mathrm{n}$ \\
\hline \multicolumn{2}{|c|}{ QoM $(3.81 \pm 0.78)$} & $4.51 \pm 0.41$ & \multicolumn{2}{|c|}{$3.00-5.17$} & $4.48 \pm 0.47$ & \multicolumn{2}{|c|}{$3.20-5.33$} & $-0.05 \pm 0.10$ & 0.616 & 51 \\
\hline \multicolumn{2}{|c|}{ Regulation $(5.0 \pm 0.82)$} & $5.65 \pm 0.76$ & \multicolumn{2}{|c|}{$4.00-7.67$} & $5.91 \pm 0.83$ & \multicolumn{2}{|c|}{$3.81-7.23$} & $0.27 \pm 0.16$ & 0.087 & 50 \\
\hline \multicolumn{2}{|c|}{ Arousal $(4.16 \pm 0.81)$} & $3.46 \pm 0.71$ & \multicolumn{2}{|c|}{$2.00-5.00$} & $4.02 \pm 0.68$ & \multicolumn{2}{|c|}{$2.29-5.43$} & $0.58 \pm 0.13$ & $<0.001$ & 51 \\
\hline \multicolumn{2}{|l|}{ B } & $\mathrm{B} \pm \mathrm{SE}$ & $\mathrm{p}$ & $\mathrm{n}$ & $\mathrm{B} \pm \mathrm{SE}$ & $\mathrm{p}$ & $\mathrm{n}$ & & & \\
\hline \multirow{3}{*}{$\frac{\pi}{4}$} & QoM & $0.00 \pm 0.04$ & 0.95 & 50 & $0.09 \pm 0.05$ & 0.080 & 46 & & & \\
\hline & Regulation & $0.00 \pm 0.07$ & 0.99 & 50 & $0.23 \pm 0.08$ & 0.006 & 46 & & & \\
\hline & Arousal & $-0.06 \pm 0.07$ & 0.42 & 50 & $-0.13 \pm 0.08$ & 0.097 & 46 & & & \\
\hline \multirow{3}{*}{$\begin{array}{l}\text { 좀 } \\
\text { D }\end{array}$} & QoM & $0.12 \pm 0.06$ & 0.06 & 50 & $0.10 \pm 0.08$ & 0.214 & 46 & & & \\
\hline & Regulation & $0.04 \pm 0.12$ & 0.74 & 50 & $0.01 \pm 0.14$ & 0.958 & 46 & & & \\
\hline & Arousal & $0.10 \pm 0.12$ & 0.40 & 50 & $0.08 \pm 0.12$ & 0.511 & 46 & & & \\
\hline
\end{tabular}

Data are given as mean $\pm S D$ and range, or parameter estimates $(B) \pm S E$, p-values and $n$.

All analyses are adjusted for gender and gestational age at delivery.

Table 5: Quality of movement (QoM), regulation and arousal at 1 week to 1 month after delivery $(\mathrm{A})$ and their association with infant erythrocyte arachidonic acid (AA) and docosahexaenoic acid (DHA) (B) 


\begin{tabular}{|c|c|c|c|}
\hline & & \multicolumn{2}{|c|}{ Infant erythrocyte $(n=44)$} \\
\hline & & AA & DHA \\
\hline \multirow{5}{*}{$\begin{array}{l}\overline{\bar{亠}} \\
\bar{\varrho} \\
\bar{\infty}\end{array}$} & Cognitive scale score & $B=-0.28 \pm 0.28, p=0.34^{1}$ & $B=-0.58 \pm 0.45, p=0.21^{2}$ \\
\hline & Receptive scale score & $B=-0.53 \pm 0.26, p=0.05^{3}$ & $B=-0.43 \pm 0.42, p=0.31^{3}$ \\
\hline & Expressive scale score & $B=-0.14 \pm 0.16, p=0.40^{4}$ & $B=-0.32 \pm 0.32, p=0.32^{5}$ \\
\hline & Fine motor scale score & $B=-0.70 \pm 0.30, p=0.03^{1}$ & $B=-0.58 \pm 0.52, p=0.27^{1}$ \\
\hline & Gross motor scale score & $B=0.19 \pm 0.22, p=0.38$ & $B=-0.44 \pm 0.42, p=0.30$ \\
\hline
\end{tabular}

Data are given as parameter estimates $(B \pm S D)$ and $p$-value $(p)$ from multiple regression analysis adjusted for ${ }^{1}$ Gender; ${ }^{2}$ Gender, corrected-age; ${ }^{3}$ Gender, maternal psychiatric disorder; ${ }^{4}$ Multiple birth; and ${ }^{5}$ Multiple birth, corrected age

Table 6: Univariate associations between infant erythrocyte arachidonic acid (AA) and docosahexaenoic acid (DHA) at 1 month of age and developmental outcomes at 1 year corrected age measured with Bayley Scales of Infant Development-III.

supplementation in preterm girls and no effect in boys $[48,49]$.

The individual variation in DHA status at a given time was substantial, but it may take a considerably larger population to detect a potential effect of DHA on development. The lack of an association between DHA and development could also be due to a lack of n-3 PUFA deficiency in the infants in the study. DHA and AA are usually measured as mass- $\%$ and "compete" with each other as well as other PUFA with respect to RBC-incorporation, it is therefore possible that the seemingly negative effect of AA to some extent is caused by reduced presence of other PUFAs. The functional domains affected by RBCAA in this study (regulation, receptive language and fine motor skills) share a relation with the periventricular midbrain structures and the cerebellum, which are where the accretion of LC-PUFA mainly occur in late gestation. DHA accumulates in the brain at a later stage than AA [4] and the impact of early sub-optimal DHA supply may only be found in later stages of neurodevelopment.

As described above, infant RBC-AA was linked to neurodevelopmental outcomes in both early and later infancy, but in an inconsistent way. We are not able to say if the early benefit and later negative effect in this study are linked or if they are due to chance, as we were unable to show an association between early neurodevelopmental maturity and psychomotor scores at 1 year, except for an association between QoM at 1 month of age and the expressive language score of BSID-III. It is possible that a larger sample size would have enabled us to demonstrate more associations between early neuromaturation and later psychomotor development.

Neurodevelopment both early in infancy and at 1 year was assessed with clinically validated tests. Our three selected NNNS summary scores (QoM, regulation and arousal) were related to what we wanted to examine (motor control, stimulus tolerance and level of alertnes) and gave reliable scores (i.e. were available in most of the infants, normally distributed and internally correlated). These functional domains also relate to brain areas associated with perinatal LC-PUFA accretion. QoM in early infancy has been addressed quite often in previous studies and is used as a measure of motor maturity in several other developmental tests (e.g., Prechtl, ENIGMA or Peabody). Regulation is a measure of the infants ability to tolerate stimulation and is closely related to habituation, which is often used as a measure of the ability to inhibit responses to a continued stimulus (tactile, visual or auditory). However, the evaluation of infant habituation in the NNNS test requires that the test is started with a sleeping infant, and in an outpatient setting such as ours, this is not always possible. BSID-III is a global developmental test, designed to contrast developmental delay and normal psychomotor development. It is considered the golden standard for developmental testing of infants and is widely used in studies of LC-PUFA influences on early childhood development, but some researchers have pointed out that it may not be ideal for detecting minor variations within normality [47,50-54].

The main limitations of the present study is the small sample size. We planned for a considerably larger study, but were not able to include more participants within the given time frame. This was mainly explained by a $40 \%$ drop in the local preterm infant delivery rate [55] due to a temporary discontinuation of a longstanding government subsidy for infertility treatment. Furthermore, the exposures (2) and outcomes (8) were tested multiple times against each other. Formal Bonferroni correction $(\mathrm{p}<0.003)$ would yield all of our tests insigificant, but the Bonferroni method is known for its very conservative approach and we found it un-suited for our explorative purpose [56]. We realize that much caution must be applied in the interpretation of our results, but they can be used for the planning future studies of LC-PUFA and infancy development. Depending on the desired outcome, and given the parameter estimates in this study, the required sample size in such studies would have to be considerably larger, e.g. 111 and 214 for QoM and arousal at 1 month of age, respectively, and 345 for BSID-III at 1 year.

Due to the observational nature of the study we cannot exclude residual confounding although we did adjust for various maternal variables, but the possibility of other confounders such as maternal stimulation of the infant or genetic influences remain. Furthermore, only $62 \%(n=33)$ were able to exclusively breastfeed and there was a highly significant difference in the frequency of maternal smokers between those still breastfeeding at 3 monts corrected age and those with a short breastfeeding duration. This is a potential bias in our study, as it resulted in a higher representation of breast-milk samples from women of higher socioeconomic status. The associations between the RBC samples and the developmental outcomes were, however, not affected by maternal smoking. There was a slight difference in maternal age between the participants and the non-participants, but this difference is probably not a clinically relevant factor. However, we did find a minor, but significant, difference in the occurrence of maternal psychiatric disorder between the infants lost to follow-up and those remaining in the study, but this were caused by only one twin mother. Maternal psychiatric disorder was a statistically significant covariate in the associations between infant LC-PUFA status and receptive language BSID-III score, but because of the small sample size, the impact of this difference is uncertain. The overall attendance at outpatient controls and follow-up was good and the consistency of our data is good. The internal validity of our study is also good as all examinations were performed by one investigator and all samples handled by one lab technician. Furthermore, we did not only rely on breast-milk samples, but also analyzed infant RBC fatty acid composition, which gives a more stable measure of LC-PUFA accretion.

\section{Conclusion}

In this relatively small cohort, late-preterm breast-milk LC-PUFA content was high compared to other preterm studies. The infants were primarily healthy with appropriate weight for gestational age and only minor developmental variations, but certain aspects of early neuromaturation and development at 1 year corrected age was associated in an inconsistent way with AA in breast-milk and infant $\mathrm{RBC}$ in the perinatal period. Our results must be approached with caution, but they warrent further testing with precise tests of sensory integrity in larger cohorts to properly investigate effects of perinatal LC-PUFA-status on early neurodevelopment in healthy late-preterm infants. 
Citation: Andersen SB, Hellgren LI, Larsen MK, Verder H, Lauritzen L (2015) Long-Chain Polyunsaturated Fatty Acids in Breast-Milk and Erythrocytes and Neurodevelopmental Outcomes in Danish Late-Preterm Infants. J Preg Child Health 2: 160. doi:10.4172/2376-127X.1000160

\section{Conflict of Interest}

None

\section{Sources of Funding}

Region Zealand Research Foundation, Foundation of Tømmerhandler Johannes Fog and Arla Foods Amba. Portable breast pumps were supplied by Medela Medical (Baar, Switzerland) and infant formula was supplied by Mead-Johnson Nutrition (Glenview, Illinois, USA)

\section{References}

1. Lauritzen L, Hansen HS, Jørgensen MH, Michaelsen KF (2001) The essentiality of long chain $\mathrm{n}-3$ fatty acids in relation to development and function of the brain and retina. Prog Lipid Res 40: 1-94.

2. P. Guesnet and JM Alessandri (2011) "Docosahexaenoic acid (DHA) and the developing central nervous system (CNS) - Implications for dietary recommendations. Biochimie 93:7-12.

3. GY Diau, AT Hsieh, EA Sarkadi Nagy, V Wijendran, PW Nathanielsz et al. (2005) "The influence of long chain polyunsaturate supplementation on docosahexaenoic acid and arachidonic acid in baboon neonate central nervous system. BMC Med 3:11.

4. Hadders-Algra M (2010) Effect of long-chain polyunsaturated fatty acid supplementation on neurodevelopmental outcome in full-term infants. Nutrients 2: $790-804$.

5. KG Sabel, C Lundqvist-Persson, E Bona, M Petzold, and B Strandvik (2009) Fatty acid patterns early after premature birth, simultaneously analysed in mothers' food, breast milk and serum phospholipids of mothers and infants. Lipids Health Dis 8:20.

6. Clandinin MT, Chappell JE, Heim T, Swyer PR, Chance GW (1981) Fatty acid utilization in perinatal de novo synthesis of tissues. Early Hum Dev 5: 355-366.

7. MT Clandinin, JE Chappell, S Leong, T Heim, PR. Swyer et al (1980) Intrauterine fatty acid accretion rates in human brain: implications for fatty acid requirements. Early Hum. Dev 4: 121-129.

8. Wells JC, Chomtho S, Fewtrell MS (2007) Programming of body composition by early growth and nutrition. Proc Nutr Soc 66: 423-434

9. Rogers I; EURO-BLCS Study Group (2003) The influence of birthweight and intrauterine environment on adiposity and fat distribution in later life. Int $\mathrm{J}$ Obes Relat Metab Disord 27: 755-777.

10. Lester BM, Tronick EZ (2004) History and description of the Neonatal Intensive Care Unit Network Neurobehavioral Scale. Pediatrics 113: 634-640.

11. Caravale B, Tozzi C, Albino G, Vicari S (2005) Cognitive development in low risk preterm infants at 3-4 years of life. Arch Dis Child Fetal Neonatal Ed 90: F474-479.

12. Lauritzen L, Jørgensen MH, Hansen HS, Michaelsen KF (2002) Fluctuations in human milk long-chain PUFA levels in relation to dietary fish intake. Lipids 37: $237-244$.

13. C. Moltó-Puigmartí, Al Castellote, X Carbonell-Estrany, and MC López-Sabater (2011) Differences in fat content and fatty acid proportions among colostrum, transitional, and mature milk from women delivering very preterm, preterm, and term infants. Clin. Nutr 30:116-123.

14. Kovács A, Funke S, Marosvölgyi T, Burus I, Decsi T (2005) Fatty acids in early human milk after preterm and full-term delivery. J Pediatr Gastroenterol Nutr 41: 454-459.

15. RJ Beijers and A Schaafsma (1996) Long-chain polyunsaturated fatty acid content in Dutch preterm breast milk; differences in the concentrations of docosahexaenoic acid and arachidonic acid due to length of gestation. Early Hum. Dev.44:215-223.

16. Lundqvist-Persson C, Lau G, Nordin P, Strandvik B, Sabel KG (2010) Early behaviour and development in breast-fed premature infants are influenced by omega-6 and omega-3 fatty acid status. Early Hum Dev 86: 407-412.

17. H. Bouwstra, D. J. Dijck-Brouwer, T. Decsi, G. Boehm, E. R. Boersma, et al., "Relationship between umbilical cord essential fatty acid content and the quality of general movements of healthy term infants at 3 months." Pediatr. Res., vol. 59, no. 5, pp. 717-22, May 2006, 16627888

18. Bouwstra H, Dijck-Brouwer DA, Wildeman JA, Tjoonk HM, van der Heide JC et al. (2003) Long-chain polyunsaturated fatty acids have a positive effect on the quality of general movements of healthy term infants. Am J Clin Nutr 78 . 313-318.

19. S. A. van Goor, D. A. Janneke Dijck-Brouwer, B. Doornbos, J. J. H. M. Erwich A. Schaafsma, et al., "Supplementation of DHA but not DHA with arachidonic acid during pregnancy and lactation influences general movement quality in 12-week-old term infants.," Br. J. Nutr., vol. 103, no. 2, pp. 235-42, Jan. 2010 19703327.

20. D. a J. Dijck-Brouwer, M. Hadders-Algra, H. Bouwstra, T. Decsi, G. Boehm, et al. "Lower fetal status of docosahexaenoic acid, arachidonic acid and essential fatty acids is associated with less favorable neonatal neurological condition.," Prostaglandins. Leukot. Essent. Fatty Acids, vol. 72, no. 1, pp. 21-8, Jan. 2005 15589396

21. El-Dib M, Massaro AN, Glass $P$, Aly H (2012) Neurobehavioral assessment as a predictor of neurodevelopmental outcome in preterm infants. J Perinato 32: 299-303.

22. Noble Y, Boyd R (2012) Neonatal assessments for the preterm infant up to 4 months corrected age: a systematic review. Dev Med Child Neurol 54: 129-139.

23. B. M. Lester, E. Z. Tronick, L. LaGasse, R. Seifer, C. R. Bauer, et al., "Summary statistics of neonatal intensive care unit network neurobehavioral scale scores from the maternal lifestyle study: a quasinormative sample.," Pediatrics, vol 113, no. 3 Pt 2, pp. 668-75, Mar. 2004, 14993525

24. L. G. Weiss, T. Oakland, and G. P. Aylward, Bayley-III Clinical Use and Interpretation, 2010. Elsevier Inc., 2010.

25. Lauritzen L, Jørgensen MH, Mikkelsen TB, Skovgaard IM, Straarup EM, et al. (2004) Maternal fish oil supplementation in lactation: effect on visual acuity and n-3 fatty acid content of infant erythrocytes. Lipids 39: 195-206.

26. FOLCH J, LEES M, SLOANE STANLEY GH (1957) A simple method for the isolation and purification of total lipides from animal tissues. J Biol Chem 226 497-509.

27. S. Hamilton, R. Hamilton, and P. Sewell, "Extraction of lipids and derivative formation." in Lipid Analysis. A Practical Approach., pp. 13-64, 1992, R. Hamilton and S. Hamilton, Eds. Oxford: IRL Press, 1992, pp. 13-64

28. T. Drachmann et al., "The source of dietary fatty acids alter the activity of secretory sphingomyelinase in the rat.," Eur. J. Lipid Sci. Technol., vol. 109(10) pp. 1003-1009, 2007.

29. Jenkins TC (2010) Technical note: common analytical errors yielding inaccurate results during analysis of fatty acids in feed and digesta samples. J Dairy Sci 93: $1170-1174$

30. Guest J, Garg M, Bilgin A, Grant R (2013) Relationship between central and peripheral fatty acids in humans. Lipids Health Dis 12: 79.

31. Stephens BE, Liu J, Lester B, Lagasse L, Shankaran S, et al. (2010) Neurobehavioral assessment predicts motor outcome in preterm infants. Pediatr 156: 366-371.

32. Boukydis CF, Bigsby R, Lester BM (2004) Clinical use of the Neonatal Intensive Care Unit Network Neurobehavioral Scale. Pediatrics 113: 679-689.

33. Scholtens S, Wijga AH, Smit HA, Brunekreef B, de Jongste JC, et al. (2009) Long-chain polyunsaturated fatty acids in breast milk and early weight gain in breast-fed infants. Br J Nutr 101: 116-121.

34. K.-G. Sabel, B. Strandvik, M. Petzold, and C. Lundqvist-Persson, "Motor mental and behavioral developments in infancy are associated with fatty acid pattern in breast milk and plasma of premature infants.," Prostaglandins. Leukot. Essent. Fatty Acids, vol. 86, no. 4-5, pp. 183-8, Apr. 2012, 22440244

35. Rodriguez A, Raederstorff D, Sarda P, Lauret C, Mendy F, et al. (2003) Preterm infant formula supplementation with alpha linolenic acid and docosahexaenoic acid. Eur J Clin Nutr 57: 727-734.

36. P. V. Pontes, A. G. Torres, N. M. F. Trugo, V. M. Fonseca, and R. Sichieri, "n-6 and $n-3$ Long-chain polyunsaturated fatty acids in the erythrocyte membrane of Brazilian preterm and term neonates and their mothers at delivery.," Prostaglandins. Leukot. Essent. Fatty Acids, vol. 74, no. 2, pp. 117-23, Feb. 2006, 16364619. 
Citation: Andersen SB, Hellgren LI, Larsen MK, Verder H, Lauritzen L (2015) Long-Chain Polyunsaturated Fatty Acids in Breast-Milk and Erythrocytes and Neurodevelopmental Outcomes in Danish Late-Preterm Infants. J Preg Child Health 2: 160. doi:10.4172/2376-127X.1000160

37. Innis SM, Nelson CM, Rioux MF, King DJ (1994) Development of visual acuity in relation to plasma and erythrocyte omega- 6 and omega- 3 fatty acids in healthy term gestation infants. Am J Clin Nutr 60: 347-352.

38. J. C. Putnam, S. E. Carlson, P. W. DeVoe, and L. a Barness, "The effect of variations in dietary fatty acids on the fatty acid composition of erythrocyte phosphatidylcholine and phosphatidylethanolamine in human infants.," Am. J. Clin. Nutr., vol. 36, no. 1, pp. 106-14, Jul. 1982, 7091020.

39. N. Auestad, D. T. Scott, J. S. Janowsky, C. Jacobsen, R. E. Carroll, et al., "Visual, Cognitive, and Language Assessments at 39 Months?: A Follow-up Study of Children Fed Formulas Containing Long-Chain Polyunsaturated Fatty Acids to 1 Year of Age," Pediatrics, vol. 112, no. 3, pp. e177-e184, 2003.

40. Smithers LG, Collins CT, Simmonds LA, Gibson RA, McPhee A, et al. (2010) Feeding preterm infants milk with a higher dose of docosahexaenoic acid than that used in current practice does not influence language or behavior in early childhood: a follow-up study of a randomized controlled trial. Am J Clin Nutr 91: 628-634.

41. S. A. Keim, J. L. Daniels, A. M. Siega-Riz, A. H. Herring, N. Dole, et al. "Breastfeeding and long-chain polyunsaturated fatty acid intake in the first 4 post-natal months and infant cognitive development: an observational study.," Matern. Child Nutr., vol. 8, no. 4, pp. 471-82, Oct. 2012, 21615865.

42. Makrides M (2013) DHA supplementation during the perinatal period and neurodevelopment: Do some babies benefit more than others? Prostaglandins Leukot Essent Fatty Acids 88: 87-90.

43. Birch EE, Garfield S, Hoffman DR, Uauy R, Birch DG (2000) A randomized controlled trial of early dietary supply of long-chain polyunsaturated fatty acids and mental development in term infants. Dev Med Child Neurol 42: 174-181.

44. D. L. O’Connor, R. Hall, D. Adamkin, N. Auestad, M. Castillo, et al., "Growth and development in preterm infants fed long-chain polyunsaturated fatty acids: a prospective, randomized controlled trial.," Pediatrics, vol. 108, no. 2, pp. 35971, Aug-2001, 11483801. pp. 359-71, Aug-2001.

45. D. T. Scott, J. S. Janowsky, R. E. Carroll, J. a Taylor, N. Auestad, et al., "Formula supplementation with long-chain polyunsaturated fatty acids: are there developmental benefits?," Pediatrics, vol. 102, no. 5, p. E59, Nov. 1998 , 9794989.
46. Meldrum SJ, D'Vaz N, Simmer K, Dunstan JA, Hird K, et al. (2012) Effects of high-dose fish oil supplementation during early infancy on neurodevelopment and language: a randomised controlled trial. Br J Nutr 108: 1443-1454.

47. Colombo J, Carlson SE, Cheatham CL, Shaddy DJ, Kerling EH, et al. (2013) Long-term effects of LCPUFA supplementation on childhood cognitive outcomes. Am J Clin Nutr 98: 403-412.

48. Makrides M, Gibson RA, McPhee AJ, Yelland L, Quinlivan J, et al. (2010) Effect of DHA supplementation during pregnancy on maternal depression and neurodevelopment of young children: a randomized controlled trial. JAMA 304: 1675-1683.

49. M. C. Allen, M. C. Allen, T. Johns, and T. Johns, "Neurodevelopmenta outcomes of preterm infants," Curr. Opin. Neurol., vol. 301, no. 2, pp. 175-182, 2008.

50. Cheatham CL, Colombo J, Carlson SE (2006) N-3 fatty acids and cognitive and visual acuity development: methodologic and conceptual considerations. Am J Clin Nutr 83: 1458S-1466S

51. J. Colombo, "Recent advances in infant cognition: implications for long-chain polyunsaturated fatty acid supplementation studies.," Lipids, vol. 36 , no. 9, pp. 919-26, Sep. 2001, 11724464

52. S. M. S. Schulzke, S. K. S. Patole, and K. Simmer, "Longchain polyunsaturated fatty acid supplementation in preterm infants ( Review )," Cochrane Libr., no. 2,2011

53. Colombo J, Carlson SE (2012) Is the measure the message: the BSID and nutritional interventions. Pediatrics 129: 1166-1167..

54. A. Beyerlein, M. Hadders-Algra, K. Kennedy, M. Fewtrell, A. Singhal, et al., "Infant formula supplementation with long-chain polyunsaturated fatty acids has no effect on Bayley developmental scores at 18 months of age--IPD metaanalysis of 4 large clinical trials.," J. Pediatr. Gastroenterol. Nutr., vol. 50, no. 1 pp. 79-84, Jan. 2010, 19881391.

55. Statistics-Denmark, "Birth rates," Statbank, 2013.

56. Levine M, Ensom MH (2001) Post hoc power analysis: an idea whose time has passed? Pharmacotherapy 21: 405-409.
Citation: Andersen SB, Hellgren LI, Larsen MK, Verder H, Lauritzen L (2015) Long-Chain Polyunsaturated Fatty Acids in Breast-Milk and Erythrocytes and Neurodevelopmental Outcomes in Danish Late-Preterm Infants. J Preg Child Health 2: 160. doi:10.4172/2376-127X.1000160
Submit your next manuscript and get advantages of OMICS Group submissions

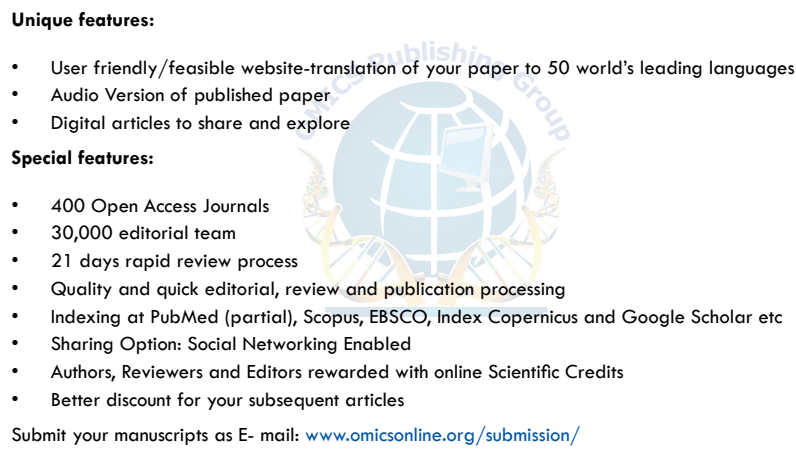

